\title{
Estudo sobre correlação do desenvolvimento da hipertensão arterial e local de trabalho em operadores da área da saúde: uma revisão integrativa
}

\author{
Study on the correlation between the development of arterial hypertension and the workplace in \\ healthcare operators: an integrative review
}

Estudio sobre la correlación entre el desarrollo de hipertensión arterial y el lugar de trabajo en operadores sanitarios: una revisión integradora

\author{
José Horácio Bairros Ramos \\ ORCID: https://orcid.org/0000-0002-8183-9507 \\ Universidade Federal do Pará, Brasil \\ E-mail: jh081970@gmail.com \\ Valéria Regina Cavalcante dos Santos \\ ORCID: https://orcid.org/0000-0002-1264-8125 \\ Universidade Federal do Pará, Brasil \\ E-mail: valregsantos@ hotmail.com
}

\begin{abstract}
Resumo
Os estudos sobre a correlação hipertensão arterial e trabalho são insuficientes para que se tenha um quadro dos riscos à que estes estão sujeitos diariamente. A revisão de literatura foi conduzida por meio de pesquisa em base de dados científicas. Objetivo: Sintetizar o que se tem na literatura acerca da correlação do ambiente de trabalho e o desenvolvimento da hipertensão arterial. Metodologia: Projetou-se a seguinte questão sobre o tema: $\mathrm{O}$ que os estudos já realizados, sobre a correlação do ambiente de trabalho, conseguiram definir sobre os efeitos deste no desenvolvimento da hipertensão arterial? Para a pesquisa desta revisão foi realizada uma busca on line nas bases de dados: CAPES, CENTRAL, SciELO, BVS, MEDLINE, Google Acadêmico, LILACS e Redalyc. Resultados: Foram selecionados 23 artigos, entre janeiro de 2016 e dezembro de 2021, que indicam que os fatores: regime terapêutico; aspectos socioeconômicos e demográficos; relação com os serviços e profissionais de saúde; aspectos psicossociais e culturais; e apoio familiar e social, incidem no desenvolvimento da hipertensão. Discussão: A hipertensão é uma doença silenciosa e de difícil diagnóstico, com uma maior prevalência em adultos, o que acarreta perda produtiva com consequências negativas para o servidor e a instituição. Conclusão: Os estudos apontam que o ambiente onde está o trabalhador, a carga e o turno de trabalho influenciam significativamente no surgimento da hipertensão arterial. Identificar o perfil de hipertensão tem como propósito planejar ações preventivas que possam melhorar estas condições.
\end{abstract}

Palavras-chave: Hipertensão arterial; Cuidado farmacêutico; Ambiente de trabalho; Tratamento da hipertensão.

\begin{abstract}
Studies on the correlation between hypertension and work are insufficient to provide a picture of the risks to which they are subject on a daily basis. The literature review was conducted through a scientific database search. Objective: To synthesize what is available in the literature about the correlation between the work environment and the development of arterial hypertension. Methodology: The following question was projected on the topic: What studies already carried out, on the correlation of the work environment, managed to define about its effects on the development of arterial hypertension? To research this review, an online search was performed in the following databases: CAPES, CENTRAL, SciELO, BVS, MEDLINE, Academic Google, LILACS and Redalyc. Results: 23 articles were selected, between January 2016 and December 2021, which indicate that the factors: therapeutic regimen; socioeconomic and demographic aspects; relationship with health services and professionals; psychosocial and cultural aspects; and family and social support, focus on the development of hypertension. Discussion: Hypertension is a silent disease and difficult to diagnose, with a higher prevalence in adults, which leads to loss of production with negative consequences for the server and the institution. Conclusion: Studies show that the environment where the worker is, the workload and the work shift significantly influence the emergence of arterial hypertension. Identifying the profile of hypertension is intended to plan preventive actions that can improve these conditions.
\end{abstract}

Keywords: Arterial hypertension; Pharmaceutical care; Desktop; Hypertension treatment. 


\begin{abstract}
Resumen
Los estudios sobre la correlación entre hipertensión y trabajo son insuficientes para ofrecer una imagen de los riesgos a los que están sujetos a diario. La revisión de la literatura se realizó mediante una búsqueda en una base de datos científica. Objetivo: Sintetizar lo disponible en la literatura sobre la correlación entre el ambiente laboral y el desarrollo de hipertensión arterial. Metodología: Se proyectó la siguiente pregunta sobre el tema: ¿Qué estudios ya realizados, sobre la correlación del ambiente laboral, lograron definir sobre sus efectos en el desarrollo de la hipertensión arterial? Para investigar esta revisión, se realizó una búsqueda en línea en las siguientes bases de datos: CAPES, CENTRAL, SciELO, BVS, MEDLINE, Academic Google, LILACS y Redalyc. Resultados: se seleccionaron 23 artículos, entre enero de 2016 y diciembre de 2021, que indican que los factores: régimen terapéutico; aspectos socioeconómicos y demográficos; relación con los servicios y profesionales de la salud; aspectos psicosociales y culturales; y apoyo familiar y social, se centran en el desarrollo de la hipertensión. Discusión: La hipertensión arterial es una enfermedad silenciosa y de difícil diagnóstico, con mayor prevalencia en adultos, lo que conlleva pérdida de producción con consecuencias negativas para el servidor y la institución. Conclusión: Los estudios muestran que el entorno en el que se encuentra el trabajador, la carga de trabajo y el turno de trabajo influyen significativamente en la aparición de hipertensión arterial. Identificar el perfil de hipertensión tiene como finalidad planificar acciones preventivas que puedan mejorar estas condiciones.
\end{abstract}

Palabras clave: Hipertención arterial; Cuidado farmacéutico; Ambiente de trabajo; Tratamiento de la hipertensión.

\title{
1. Introdução
}

Ao longo do tempo, as mudanças no padrão de morbimortalidade culminaram com substituição de uma maior prevalência das doenças infecto-parasitárias pelas doenças crônico-degenerativas. Essa modificação conduziu as doenças crônicas não transmissíveis (DCNT) ao patamar de problema de saúde mundial, constituindo uma ameaça à saúde e ao desenvolvimento humano (Barreto, et al., 2017).

O estado de saúde de um indivíduo pode ser influenciado pelo meio em que vive, por suas relações sociais, bem como por suas condições sócio-econômico-culturais, sendo precisamente indicada por sinais fisiológicos, entre eles a pressão arterial, cuja aferição deve ser feita por profissionais de saúde treinados e em toda avaliação clínica.

Os determinantes da saúde são frequentemente agrupados de acordo com a literatura consultada, com maior ou menor número de categorias. Os determinantes fixos ou biológicos, de que é exemplo à idade, sexo e fatores genéticos; os determinantes econômicos e sociais, de que é exemplo à posição, o estrato social, o emprego, a pobreza, a exclusão social; os ambientais, tais como a qualidade do ar e da água, ambiente social; os de estilos de vida, sendo a alimentação, atividade física, tabagismo, álcool e comportamento sexual alguns exemplos. Incluem-se ainda o acesso aos serviços, como educação, saúde, serviços sociais, transportes e lazer (Carrapato, et al., 2017).

Hipertensão (pressão arterial elevada) é um fator de risco importante para doenças cardiovasculares, tais como doença coronária, acidente vascular cerebral e insuficiência cardíaca. Reduzir a pressão arterial para níveis normais é eficaz na redução dos riscos dessas doenças. Muitos dos fatores de risco relacionados com a hipertensão, tais como sedentarismo, dieta rica em sal e gordura ou tabagismo, estão relacionados com o estilo de vida. A atividade física é reconhecida como um componente essencial de um estilo de vida saudável. No entanto, muitas pessoas podem ter dificuldade em praticar exercício que se encaixe nas suas atividades da vida diária (Lee, et al., 2013).

O Instituto Brasileiro de Geografia e Estatística (IBGE) em parceria com o Ministério da Saúde (MS) apontam que a Atenção Primária, promovida pelo Sistema Único de Saúde (SUS) é referência de atendimento para os brasileiros que possuem doenças crônicas não transmissíveis. O estudo levantou que 24\% dos indivíduos alegaram diagnóstico de hipertensão em 2019, sendo essa a mais frequente entre as doenças crônicas, com 38,1 milhões de pessoas nessa condição. Desses, 72,2\% afirmaram ter recebido assistência médica para hipertensão há menos de um ano no país e 66,4\% deles haviam feito a última consulta no SUS. Os postos de saúde foram as unidades mais citadas $(46,6 \%)$ pelos pacientes na procura por consultas, Ministério da Saúde/Saúde e Vigilância Sanitária/Pesquisa Nacional de Saúde [MS/SVS/PNS] (2020).

Dados da Pesquisa de Vigilância de Fatores de Risco e Proteção para Doenças Crônicas de 2017 (VIGITEL), do 
Ministério da Saúde, aponta que 20,7\% da população de Belém (PA) tem diagnóstico médico de hipertensão arterial. Entre as pessoas da capital a doença atinge $23,4 \%$ de mulheres, enquanto $17,5 \%$ são homens. O percentual da população geral permaneceu estável. De acordo com o estudo, $24,3 \%$ da população das capitais do país se referiram com a conhecida "pressão alta". As mulheres, ainda, continuam com maior índice em relação aos homens, tendo registrado $26,4 \%$ contra $21,7 \%$ deles, Ministério da Saúde/ Saúde e Vigilância Sanitária/Agência Saúde [MS/SVS/AS] (2018).

Para a prevenção da doença, o Ministério da Saúde recomenda que a população adote alguns hábitos saudáveis, como a prática de atividade física regular e uma alimentação com baixo teor de sal. Para o tratamento, o Sistema Único de Saúde (SUS) oferece gratuitamente medicamentos nas Unidades Básicas de Saúde (UBS) e pelo programa Farmácia Popular (MS/SVS/AS, 2018).

Considera-se que a adesão ao tratamento da hipertensão arterial é fundamental para que o paciente tenha melhor qualidade de vida e menor risco de complicações cardiovasculares. Em decorrência, entende-se que manter a pressão arterial sistêmica sob controle constitui-se num indicador do cumprimento das prescrições recomendadas pelo médico e equipe de saúde. Na intenção de identificar a melhor forma de promover a adesão ao tratamento destes pacientes, delimitou-se como objetivo para o presente estudo identificar, na literatura, a correlação do trabalho exercido e o surgimento da hipertensão arterial sistêmica.

\subsection{Hipertensão Arterial e seu Diagnóstico}

A hipertensão arterial (HA) é uma doença crônica não transmissível definida por níveis pressóricos, em que os benefícios do tratamento (não medicamentoso e/ou medicamentoso) superam os riscos (Barroso, et al., 2020).

Trata-se de uma condição multifatorial, que depende de fatores genéticos/epigenéticos, ambientais e sociais, caracterizada por elevação persistente da pressão arterial (PA), ou seja, PA sistólica (PAS) maior ou igual a $140 \mathrm{mmHg}$ e/ou PA diastólica (PAD) maior ou igual a $90 \mathrm{mmHg}$, medida com a técnica correta, em pelo menos duas ocasiões diferentes, na ausência de medicação anti-hipertensiva (Barroso et al., 2020). A cada 20 milímetros de mercúrio (mmHg) do valor elevado da pressão sistólica e $10 \mathrm{mmHg}$ da diastólica, dobra-se o risco de morte por acidente vascular encefálico (AVE) e outras doenças cardíacas e vasculares (Benjamin, et al., 2018).

A hipertensão é uma condição de longo prazo em que a pressão arterial está elevada. É a principal causa de morte em todo o mundo, afetando mais de 1,4 bilhão de pessoas e sendo responsável por mais de 28.000 mortes por dia. Inicialmente, não causa nenhum sintoma, mas se não for tratada, pode causar derrame, ataque cardíaco, doença renal, perda de visão e demência. O controle da pressão arterial elevada pode ajudar a proteger contra essas condições e há muitas medidas que podem ser tomadas para ajudar a reduzir a pressão arterial, International Society of Hypertension [ISH] (2018).

O tratamento adequado da hipertensão arterial reduz o risco cardiovascular para níveis normais. No entanto, o maior problema para o controle da hipertensão é a adesão ao tratamento. Apesar dos tratamentos muito eficazes e econômicos, os níveis-alvo de pressão arterial nem sempre são alcançados, mesmo em países onde o custo da medicação não é um problema, International Society of Hypertension [ISH] (2018).

No Brasil estima-se que a prevalência de hipertensão arterial pode chegar até $24,7 \%$. Um fator agravante é que, por ser uma doença silenciosa, não manifesta sintomas na maioria das pessoas, tornando mais difícil seu diagnóstico, Ministério da Saúde/ Secretaria de Vigilância em Saúde/Departamento de Análise em Saúde e Vigilância de Doenças Não Transmissíveis [MS/SVS/DASVDNT] (2019).

Por se tratar de condição frequentemente assintomática, a hipertensão costuma evoluir com alterações estruturais e/ou funcionais em órgãos-alvo, como coração, cérebro, rins e vasos. Ela é o principal fator de risco modificável com associação independente, linear e contínua para doenças cardiovasculares (DCV), doença renal crônica (DRC) e morte prematura. 
Associa-se a fatores de risco metabólicos para as doenças dos sistemas cardiocirculatório e renal, como dislipidemia, obesidade abdominal, intolerância à glicose, e diabetes melito (DM) (Forouzanfar, et al., 2017; Carey, et al., 2018).

Diagnosticar hipertensão é relativamente fácil, mas depende de um estudo que engloba vários fatores, desde o surgimento de cefaleia e dor de cabeça, principalmente na região da nuca, até medidas antropométricas e resultado de exames laboratoriais, clínicos e medições pressóricas desvinculadas do uso de medicamentos antihipertensivos.

Os exames mais comumente solicitados pelo cardiologista para fazer uma análise da condição pressórica do paciente e poder concluir o estudo da hipótese diagnóstica (HD) são a tomografia cardíaca computadorizada, ultrassom das carótidas, exame laboratorial de proteína $\mathrm{C}$ reativa, dosagem de lipoproteína (a), e monitorização ambulatoria da pressão arterial MAPA (Scala, et al., 2015; Buuren, et al., 2017).

\section{Tomografia cardíaca computadorizada}

A tomografia cardíaca computadorizada é um teste de imagem indolor que usa raios-X para obter imagens detalhadas do coração e seus vasos sanguíneos. Os computadores são capazes de combinar essas imagens para criar um modelo tridimensional do coração e detectar ou avaliar doenças coronárias, acúmulo de cálcio nas artérias, problemas com a aorta, com a função cardíaca ou outras alterações que podem estar relacionadas ao aumento da pressão arterial. O preparo pode incluir o uso de medicamentos para diminuir frequência cardíaca ou o de um contraste colorido para destacar os vasos sanguíneos.

\section{Ultrassom das carótidas}

Este é um exame fácil e indolor para avaliar o interior das artérias carótidas, que transportam oxigênio ao cérebro. Em pessoas com colesterol alto ou pressão alta, é comum que essas artérias apresentem placas de gordura em suas paredes. Essas placas podem se acumular a ponto de interromper a passagem de sangue ou se romper, formando um coágulo que, se transportado ao cérebro, pode provocar um Acidente Vascular Cerebral (AVC). O exame é bastante simples e não exige preparo: a pessoa fica deitada enquanto o técnico passa o aparelho de ultrassom pelas laterais do pescoço, com o auxílio de um gel.

\section{Proteína C reativa}

A proteína C-reativa é encontrada no sangue quando existe alguma inflamação no corpo, o que pode contribuir para a elevação da pressão arterial, causando alterações nas células que revestem as paredes dos vasos sanguíneos. Um simples exame de sangue pode identificar quando há excesso dessa proteína no organismo e servir de alerta para que se busque a origem da inflamação.

\section{Dosagem de lipoproteína (a)}

A lipoproteína (a) é similar ao colesterol LDL, conhecido por ser o colesterol (bom ou ruim) e também pode causar o entupimento das artérias, quando encontrada em níveis maiores do que $30 \mathrm{mg} / \mathrm{dl}$, sendo, portanto, um fator de risco importante para doenças cardiovasculares. Esse também é um exame simples, feito a partir da coleta de sangue.

\section{Monitorização Ambulatorial da Pressão Arterial (MAPA)}

A monitorização ambulatorial da pressão arterial é um exame que mede a pressão a cada 20 minutos, durante 24 horas, e permite analisá-la não somente durante a vigília e o sono, mas também durante possíveis sintomas como tontura, dor no peito e desmaio. Para a realização desse exame, é colocado um monitor leve e pequeno na cintura, conectado a um tubo fino de plástico a uma braçadeira. A cada 20 minutos o monitor insufla a braçadeira e registra a pressão. Após 24 horas, os dados 
coletados geram um gráfico das pressões registradas. É usual que se peça ao paciente que tome nota dos horários em que dormiu, acordou, almoçou, jantou, sentiu algum sintoma, fez atividades físicas ou teve algum acontecimento importante durante o dia.

\section{Metodologia}

Decidiu-se pela revisão integrativa da literatura, definida como um método que permite a síntese de conhecimento e a incorporação da aplicabilidade de resultados de estudos significativos na prática (Souza, 2010). É objetivo apresentar os conhecimentos gerais e específicos com base na mais recente evidência científica. A síntese viabiliza a contextualização do pesquisador acerca da temática, além de sinalizar lacunas do conhecimento que precisam ser preenchido com novos estudos, contribuindo para discussões a respeito dos métodos e resultado de pesquisas anteriores (Polit, et al., 2006).

Para a elaboração da revisão integrativa, no primeiro momento o revisor determina o objetivo específico, formula os questionamentos a serem respondidos e, então, realiza a busca para identificar e coletar o máximo de pesquisas primárias relevantes dentro dos critérios de inclusão e exclusão previamente estabelecidos (Beyea, 1998).

Desta forma, para nortear a realização do presente estudo, formulou-se a seguinte questão: O que os estudos já realizados, sobre a correlação do ambiente de trabalho, conseguiram definir sobre os efeitos deste no desenvolvimento da hipertensão arterial?

Para a pesquisa desta revisão, feita nos meses de outubro, novembro e dezembro de 2021, foi realizada uma busca on line nas bases de dados: Portal de Periódicos da Coordenação de Aperfeiçoamento de Pessoal de Nível Superior (CAPES), Cochrane Central Register of Controlled Trials (CENTRAL), Scientific Eletrônic Library Online (SciELO), Biblioteca Virtual em Saúde (BVS), Medical Literature Analysis and Retrieval System Online (MEDLINE), Google Acadêmico, Literatura Latino-Americana e do Caribe em Ciências da Saúde (LILACS) e Red de Revistas Científicas de América Latina y el Caribe, España y Portugal (Redalyc). A escolha por essas bases de dados deve-se ao fato de englobarem publicações nacionais e internacionais já validadas e certificadas.

Os descritores utilizados para a busca foram: hipertensão arterial; cuidado farmacêutico; ambiente de trabalho; e tratamento da hipertensão, nos idiomas português, inglês e espanhol; e realizou-se o cruzamento entre eles. Os critérios de inclusão definidos para o estudo foram: artigos voltados para a questão norteadora; ser publicado entre o período de 2016 e 2021; em um dos idiomas (português, inglês e espanhol) e estar disponibilizado na íntegra.

Realizou-se a leitura dos artigos para confirmação de que contemplavam os tópicos necessários para o embasamento da temática envolvida. Por conveniência do estudo, as publicações de teses, dissertações ou monografias não foram consideradas devido à limitação do acesso ao material. Para seleção do artigo foi considerado aquele que no título estava expressamente clara uma associação entre as palavras "hipertensão arterial", "cuidado farmacêutico" "ambiente de trabalho" e "tratamento da hipertensão".

Chegou-se a 79 artigos, sendo utilizados 23 para compor a revisão, tendo cinco sendo excluídos, pois sua publicação original é anterior aos anos delimitados para análise e oito por não serem específicos ao tema proposto, apesar de contarem com descritores abrangentes ao mesmo.

A seguir, identificou-se a força da evidência adotada para a elaboração da revisão integrativa do presente estudo: tipo I - Evidência forte a partir de pelo menos uma publicação de revisão sistemática de múltiplos experimentos controlados randomizados, bem delineados; tipo II - Evidência forte a partir de pelo menos uma publicação de experimento controlado, randomizado, corretamente projetado, com tamanho apropriado e em cenário clínico apropriado; tipo III - Evidência a partir de apenas um experimento bem delineado, sem randomização, de apenas um grupo do tipo antes e depois, de coorte, de séries 
temporais, ou de estudos caso-controle; tipo IV - Evidência a partir de estudos não experimentais por mais de um centro ou grupo de pesquisa; e tipo V - Opiniões de autoridades respeitadas, baseadas em evidência clínica, estudos descritivos ou relatórios de comitês de especialistas (Pereira, et al., 2006).

Quadro 1 - Apresentação e caracterização dos artigos selecionados dos agregadores de base de dados Portal de Periódicos CAPES, REDALYC e EBSCO, e que apresentam as ações desenvolvidas para promover a qualidade de vida de pessoas com hipertensão.

\begin{tabular}{|c|c|c|c|c|c|}
\hline $\mathrm{N}^{\circ}$ & ANO & $\begin{array}{c}\text { Base de dados / } \\
\text { Força }\end{array}$ & Autor(es) & Título & Periódico \\
\hline 1 & 2016 & $\begin{array}{l}\text { SciELO } \\
\text { II }\end{array}$ & $\begin{array}{c}\text { Pimenta, A.M., \& } \\
\text { Assunção, A.A. (2016) }\end{array}$ & $\begin{array}{l}\text { Estresse no trabalho e hipertensão } \\
\text { arterial em profissionais de } \\
\text { enfermagem da rede municipal de } \\
\text { saúde de Belo Horizonte, MG, } \\
\text { Brasil }\end{array}$ & Ver Bras Saude Ocup 2016;41:e61/11 \\
\hline 2 & 2017 & $\begin{array}{l}\text { SciELO } \\
\text { II }\end{array}$ & $\begin{array}{c}\text { Carvalho, A.C.S., } \\
\text { Maçaneiro, ALS, } \\
\text { Santos, E.T.S., Macedo, } \\
\text { G.C., Silva, S.B., Flores, } \\
\text { G.R., \& Cubo, R.C.P. } \\
\text { (2017) }\end{array}$ & $\begin{array}{l}\text { Estresse do Enfermeiro no } \\
\text { Ambiente de Trabalho e Evolução } \\
\text { para Hipertensão Arterial }\end{array}$ & $\begin{array}{c}\text { Anais da } 12^{a} \text { Semana Acadêmica de } \\
\text { Enfermagem das FISA/FUNEC: Boas } \\
\text { práticas de Enfermagem e a construção } \\
\text { de uma sociedade democrática / v.3, } \\
\text { n.3, 2017. ISSN 2525-8141. }\end{array}$ \\
\hline 3 & 2017 & $\begin{array}{l}\text { Redalyc } \\
\text { II }\end{array}$ & $\begin{array}{c}\text { Malacarne, F.S., } \\
\text { Prudente, R.C.S., \& } \\
\text { Fonseca, M.R.C. (2017) }\end{array}$ & $\begin{array}{l}\text { Shift work: repercussions on } \\
\text { health among workers of an } \\
\text { Electricity Distribution Company }\end{array}$ & $\begin{array}{c}\text { Perspectivas Médicas, } 28(2): 30-36, \\
\text { mai/ago } 2017 . \\
\text { DOI:10.6006/perspectmed.02042017.5 } \\
842316589\end{array}$ \\
\hline 4 & 2019 & $\begin{array}{l}\text { SciELO } \\
\text { III }\end{array}$ & $\begin{array}{l}\text { Neto, E.M.N., Tania, } \\
\text { M.A., \& Sousa, C.C. } \\
\qquad(2019)\end{array}$ & $\begin{array}{l}\text { Hipertensão Arterial e Diabetes } \\
\text { Mellitus entre trabalhadores da } \\
\text { saúde: associação com hábitos de } \\
\text { vida e estressores ocupacionais }\end{array}$ & $\begin{array}{c}\text { Revista Brasileira de Saúde } \\
\text { Ocupacional ISSN: 2317-6369. } \\
\text { http://dx.doi.org/10.1590/2317- } \\
6369000034218 \\
\end{array}$ \\
\hline 5 & 2019 & $\begin{array}{l}\text { SciELO } \\
\text { I }\end{array}$ & $\begin{array}{l}\text { Nascimento, J.O.V., } \\
\text { Santos, J., Meira, K.C., } \\
\text { Pierin, A.M.G., \& } \\
\text { Souza-Talarico, J.N. } \\
\text { (2019). }\end{array}$ & $\begin{array}{l}\text { Shift work of nursing professional } \\
\text { and blood pressure, burnout and } \\
\text { common mental disorders. }\end{array}$ & $\begin{array}{l}\text { Ver Esc Enferm USP. 2019;53:e03443. } \\
\text { DOI: http://dx.doi.org/10.1590/S1980- } \\
\text { 220X2018002103443 }\end{array}$ \\
\hline 6 & 2019 & $\begin{array}{l}\text { MEDLINE } \\
\text { I }\end{array}$ & $\begin{array}{l}\text { Domingues, J.G, Silva, } \\
\text { B.B.C., Bierhals, I.O., \& } \\
\text { Barros, F.C (2019) }\end{array}$ & $\begin{array}{l}\text { Noncommunicable } \text { diseases } \\
\text { among nursing professionals at a } \\
\text { charitable hospital in Southern } \\
\text { Brazil }\end{array}$ & $\begin{array}{c}\text { Epidemiol.Serv.Saúde vol.28 no.2 } \\
\text { Brasília June2019 EpubJuly04, } \\
\text { http://dx.doi.org/10.5123/s1679- } \\
\text { 49742019000200011 } \\
\end{array}$ \\
\hline 7 & 2020 & $\begin{array}{l}\text { MEDLINE } \\
\text { III }\end{array}$ & $\begin{array}{l}\text { Vieira, M.R.M., } \\
\text { Magalhães, T.A., Silva, } \\
\text { R.R.V., Vieira, M.M., } \\
\text { Paula, A.M.B., Araújo, } \\
\text { V.B., Ferreira, E.F., \& } \\
\text { Haikal, D.S. (2020) }\end{array}$ & $\begin{array}{l}\text { Hipertensão Arterial e trabalho } \\
\text { entre docentes da educação básica } \\
\text { da rede pública de ensino }\end{array}$ & $\begin{array}{c}\text { Ciência \& Saúde Coletiva, 25(8):3047- } \\
\text { 3061, 2020 - } \\
\text { https://doi.org/10.1590/1413- } \\
81232020258.26082018\end{array}$ \\
\hline 8 & 2020 & $\begin{array}{l}\text { SciELO } \\
\text { II }\end{array}$ & $\begin{array}{l}\text { Xavier, P.B., Garcez, A., } \\
\text { Cibeira, G.H., Germano, } \\
\text { A., \& Olinto, M.T.A. } \\
\text { (2020) }\end{array}$ & $\begin{array}{l}\text { Fatores Associados à Ocorrência } \\
\text { de Hipertensão Arterial em } \\
\text { Trabalhadores da Indústria do } \\
\text { Estado do Rio Grande do Sul, } \\
\text { Brasil }\end{array}$ & $\begin{array}{l}\text { Arq. Bras. Cardiol. } 117 \text { (3) • Set } 2021 \bullet \\
\text { https://doi.org/10.36660/abc.20190815 }\end{array}$ \\
\hline 9 & 2020 & $\begin{array}{l}\text { LILACS } \\
\quad \text { I }\end{array}$ & $\begin{array}{l}\text { Ribeiro, V.E.R., \& } \\
\text { Fernandes, R.C.P. } \\
\text { (2020) }\end{array}$ & 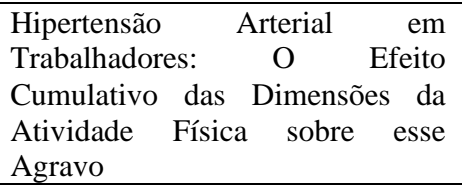 & $\begin{array}{l}\text { Arq. Bras. Cardiol. } 114 \text { (5) • } \\
\text { Maio 2020 • } \\
\text { https://doi.org/10.36660/abc.20190065 }\end{array}$ \\
\hline 10 & 2021 & $\begin{array}{l}\text { EBSCO } \\
\text { Information } \\
\text { Services } \\
\text { III }\end{array}$ & $\begin{array}{c}\text { Santiago, C.D.S., } \\
\text { Travassos, M.C.P., } \\
\text { Sousa, A.M., Almeida, } \\
\text { G.S., \& Toledo, N.N. } \\
\text { (2021) }\end{array}$ & $\begin{array}{l}\text { Pressão Arterial Elevada em } \\
\text { Servidores de Universidades } \\
\text { Públicas no Norte do Brasil }\end{array}$ & $\begin{array}{l}\text { Revista Cogitare Enfermagem. 2021, } \\
\text { Vol. 26, p986-1005. 20p. } \\
\text { https://revistas.ufpr.br/cogitare/article/v } \\
\text { iew/74371 }\end{array}$ \\
\hline
\end{tabular}

Fonte: Ramos (2021). 


\section{Resultados}

Nas bases de dados consultadas foram encontrados 79 artigos. Após leitura e análise dos artigos, baseado nos critérios de inclusão e exclusão e, tendo como orientação os descritores propostos para a seleção foram catalogados e identificados os que subsidiam esta revisão.

Chegou-se a um total de 23 artigos selecionados, dos quais seis são no idioma inglês e dezessete em português. O estudo predominante foi o transversal e abrangeu artigos publicados entre os anos de 2016 e 2021. Os artigos encontrados no idioma espanhol foram encontrados, também, no idioma português. Do total de artigos encontrados cinco foram descartados pelo fato de que sua publicação original foi anterior aos anos delimitados para análise e oito por não serem específicos ao tema proposto, apesar de contarem com descritores abrangentes ao mesmo.

A partir dos estudos feitos foram identificados os principais fatores que influenciam no surgimento ou agravamento da hipertensão arterial, destacando-se a insatisfação com o trabalho desenvolvido, excesso de peso ou quadro de obesidade, trabalhar no horário noturno, baixa renda, idade entre 30 e 45 anos. Foi observado que nos trabalhos onde o segmento feminino se faz mais presente, enfermagem e educação, os estudos apontam que as mulheres desenvolvem hipertensão com maior frequência. Enquanto que nas atividades tipicamente masculinas o inverso se faz presente. Mas não se verificou esta importante observação nos estudos.

As evidências encontradas nos artigos observados mostram, ainda, a correlação existente entre o trabalho desenvolvido e o desenvolvimento da hipertensão arterial. Importante destacar que o estudo dessas evidências permitem uma avaliação crítica e que sustenta os resultados da pesquisa, além de embasarem a prática baseada nestas evidências.

Nos estudo sobre o estresse no trabalho e hipertensão arterial em profissionais de enfermagem da rede municipal de Belo Horizonte-MG (Pimenta, et al., 2016); estresse do enfermeiro no ambiente de trabalho e evolução para hipertensão arterial (Carvalho, et al., 2017); shift work of nursing professional and blood pressure, burnout and common mental disorders (Nascimento, et al., 2019); noncommunicable diseases among nursing professional at a charitable hospital in Southern Brazil (Gonçalves, et al., 2019), os pesquisadores correlacionaram atividade laboral e desenvolvimento ou agravamento do quadro de hipertensão arterial associados a hábitos alimentares não saudáveis. Deve-se, observar, ainda, que por se tratar de ambiente hospitalar a prevalência do segmento feminino na atividade de enfermagem é maior que do segmento masculino.

Nos dois estudos realizados com profissionais da educação, um deles com professores da educação básica (Vieira et al., 2020); e outro com servidores de universidade (Santiago, et al., 2021), ficou evidente a correlação da situação de trabalho com estressores ocupacionais para o desenvolvimento da hipertensão arterial. Destaca-se nestes estudos que os professores mais velhos e com sobrepeso ou obesidade, renda de até 3 salários mínimos e insatisfeitos com seu trabalho apresentaram maior taxa de desenvolvimento de hipertensão.

Nos estudos que envolvem trabalhadores de uma empresa de energia (Malacarne, et al., 2017); trabalhadores de uma indústria no Rio Grande do Sul (Xavier, et al., 2020); e com trabalhadores da limpeza urbana e indústria de calçados na Bahia (Ribeiro, et al., 2020), observou-se que o desenvolvimento da hipertensão se mostrou maior no segmento masculino, com faixa etária entre 30 e 45 anos, portadores de excesso de peso ou já com obesidade. Enquanto que o segmento feminino, de melhor condição econômica manteve seus níveis dentro dos padrões clínicos. 
Quadro 2 - Síntese dos artigos selecionados para a revisão de literatura de acordo com autor/ano, tipo de estudo, principais resultados e conclusão.

\begin{tabular}{|c|c|c|c|}
\hline Autor/Ano & Tipo de estudo & $\begin{array}{l}\text { Principais resultados } \\
\end{array}$ & \begin{tabular}{|c|} 
Conclusões \\
\end{tabular} \\
\hline $\begin{array}{l}\text { Pimenta, A.M., \& } \\
\text { Assunção, A.A. } \\
\text { (2016) }\end{array}$ & Transversal & $\begin{array}{l}\text { Dos profissionais estudados, } 42,1 \% \text { eram enfermeiros e } \\
57,8 \% \text {, técnicos ou auxiliares de enfermagem. A hipertensão } \\
\text { foi diagnosticada em } 16,9 \% \text {. Sexo feminino }(\mathrm{RP}=0,56) \text {, } \\
\text { avançar da idade (p de tendência linear }<0,001) \text {, renda } \\
\text { familiar de } 4 \text { ou mais salários mínimos }(\mathrm{RP}=0,39) \text { e o } \\
\text { estresse no trabalho (RP }=2,53) \text { permaneceram } \\
\text { independentemente associadas à HA após o ajuste } \\
\text { multivariado dos dados. }\end{array}$ & $\begin{array}{l}\text { A prevalência da HA entre os trabalhadores da equipe de } \\
\text { enfermagem da rede municipal de saúde de Belo } \\
\text { Horizonte (MG) foi de } 16,9 \% \text {. No que diz respeito às } \\
\text { características demográficas e socioeconômicas dos } \\
\text { profissionais da equipe de enfermagem, verificou-se que a } \\
\text { maioria era do sexo feminino }(90,5 \%) \text {, tinha entre } 20 \text { e } 49 \\
\text { anos de idade }(80,6 \%) \text {, cor de pele não branca }(64,5 \%) \text {, } \\
\text { era casada/união estável }(51,7 \%) \text { e ganhava até } 4 \text { salários } \\
\text { mínimos ( } 70 \%) \text {. Ademais, as seguintes frequências de } \\
\text { hábitos de vida estavam presentes entre eles: } 11 \% \text { de } \\
\text { tabagismo; } 5,4 \% \text { de dependência alcoólica; e } 55,7 \% \text { de } \\
\text { sedentarismo. }\end{array}$ \\
\hline $\begin{array}{l}\text { Carvalho, A.C.S., } \\
\text { Maçaneiro, ALS, } \\
\text { Santos, E.T.S., } \\
\text { Macedo, G.C., } \\
\text { Silva, S.B., Flores, } \\
\text { G.R., \& Cubo, } \\
\text { R.C.P. (2017) }\end{array}$ & Transversal & $\begin{array}{l}\text { A falta de trabalhadores de enfermagem tem levado à } \\
\text { sobrecarga dos profissionais, ao mesmo tempo em que a } \\
\text { divisão técnica do trabalho e a relação conflitante com } \\
\text { outras categorias do setor de saúde têm diminuído a } \\
\text { autonomia e o poder decisório sobre o seu fazer, dessa } \\
\text { forma, os profissionais de enfermagem são muito } \\
\text { vulneráveis ao estresse no trabalho. }\end{array}$ & $\begin{array}{l}\text { Concluiu-se que a relação entre o estresse no trabalho e a } \\
\text { HA em profissionais de enfermagem demonstra que as } \\
\text { condições psicossociais aumentam a vulnerabilidade } \\
\text { desses trabalhadores à ocorrência de uma doença de } \\
\text { grande importância para a Saúde Pública brasileira: a } \\
\text { hipertensão arterial. }\end{array}$ \\
\hline $\begin{array}{l}\text { Malacarne, F.S., } \\
\text { Prudente, R.C.S., \& } \\
\text { Fonseca, M.R.C. } \\
\text { (2017) }\end{array}$ & Transversal & $\begin{array}{l}\text { Não houve associação estatística entre hábitos de saúde, } \\
\text { antecedentes mórbidos, classificação do IMC e o turno de } \\
\text { trabalho, porém observou-se tendência de obesidade entre os } \\
\text { trabalhadores noturnos, quando comparados aos } \\
\text { trabalhadores diurnos (OR: } 3,22 ; \text { IC: } 1,02-10,15 ; p=0,082) \text {, } \\
\text { ainda que rigorosamente não tenha apresentado significância } \\
\text { estatística. }\end{array}$ & $\begin{array}{l}\text { A síndrome metabólica (SM) é um agrupamento de } \\
\text { distúrbios que inclui resistência à insulina, obesidade } \\
\text { central, elevados níveis de triglicerídeos, baixos níveis de } \\
\text { HDL e hipertensão arterial, e está baseada na presença de } \\
\text { três ou mais destes componentes em um mesmo } \\
\text { indivíduo. O trabalho noturno é apontado como fator de } \\
\text { risco para o desenvolvimento de SM, bem como, para o } \\
\text { risco cardiovascular, porém, neste estudo não se observou } \\
\text { diferenças significativas entre turno de trabalho e } \\
\text { dislipidemia, diabetes mellitus e hipertensão arterial. }\end{array}$ \\
\hline $\begin{array}{l}\text { Neto, E.M.N., } \\
\text { Tania, M.A., \& } \\
\text { Sousa, C.C. (2019) }\end{array}$ & Transversal & $\begin{array}{l}\text { A prevalência de HAS e DM entre os trabalhadores foram } \\
\text { de } 18,0 \% \text { e } 4,0 \% \text {, respectivamente. Após o ajuste por sexo e } \\
\text { idade, o trabalho passivo associou-se à HAS e ao DM, e o } \\
\text { tabagismo associou-se apenas ao DM. }\end{array}$ & $\begin{array}{l}\text { O hábito de fumar e o trabalho realizado sob condições } \\
\text { psicossociais desfavoráveis (trabalho passivo) devem } \\
\text { receber atenção especial na prevenção de HAS e DM. É } \\
\text { necessário estimular a adoção de hábitos de vida } \\
\text { saudáveis e de condições de trabalho adequadas para } \\
\text { prevenir o adoecimento crônico e proteger a saúde dos } \\
\text { trabalhadores. }\end{array}$ \\
\hline $\begin{array}{l}\text { Nascimento, J.O.V., } \\
\text { Santos, J., Meira, } \\
\text { K.C., Pierin, } \\
\text { A.M.G., \& Souza- } \\
\text { Talarico, J.N. } \\
\text { (2019). }\end{array}$ & Transversal & $\begin{array}{l}\text { A média de idade dos profissionais foi de } 39,6 \text { anos, a } \\
\text { maioria era do sexo feminino }(82,7 \%) \text {, da raça/cor não } \\
\text { branca }(54,5 \%) \text { e vivia com companheiro }(70,6 \%) \text {. Os } \\
\text { pesquisados dormiam em média } 6 \text { horas diárias, e as } \\
\text { comorbidades mais referidas foram dislipidemias }(28,1 \%) \text { e } \\
\text { hipertensão arterial }(25,5 \%)\end{array}$ & $\begin{array}{l}\text { Os trabalhadores de enfermagem que atuavam em turnos } \\
\text { apresentaram maior prevalência de fatores negativos } \\
\text { relacionados ao trabalho, hábitos e estilos de vida } \\
\text { inadequados, além de alteração da pressão no período de } \\
\text { sono, quando comparados aos trabalhadores que não } \\
\text { estavam expostos a essa escala de trabalho. }\end{array}$ \\
\hline $\begin{array}{l}\text { Domingues, J.G, } \\
\text { Silva, B.B.C., } \\
\text { Bierhals, I.O., \& } \\
\text { Barros, F.C (2019) }\end{array}$ & Transversal & $\begin{array}{l}\text { Do total de } 272 \text { funcionários avaliados, } 29,4 \% \text { afirmaram ter } \\
\text { DCNT, 48,9\% faziam uso de medicamento contínuo e } \\
73,9 \% \text { referiram antecedentes familiares para DCNT; dos } \\
\text { entrevistados, 20,6\% usavam anti-hipertensivos, e destes, } \\
27,7 \% \text { apresentaram níveis tensionais elevados e } 2,6 \% \\
\text { referiram usar hipoglicemiantes; destes últimos, } 42,9 \% \\
\text { apresentaram níveis glicêmicos elevados. }\end{array}$ & $\begin{array}{l}\text { Dos profissionais autodeclarados hipertensos e diabéticos, } \\
\text { aqueles em uso de medicações apresentaram valores } \\
\text { tensionais e glicemia mais elevados do que os que não } \\
\text { faziam tratamento medicamentoso. }\end{array}$ \\
\hline $\begin{array}{l}\text { Vieira, M.R.M., } \\
\text { Magalhães, T.A., } \\
\text { Silva, R.R.V., } \\
\text { Vieira, M.M., Paula, } \\
\text { A.M.B., Araújo, } \\
\text { V.B., Ferreira, E.F., } \\
\text { \& Haikal, D.S. } \\
\text { (2020) }\end{array}$ & Transversal & $\begin{array}{l}\text { A prevalência de HA foi de } 25 \% \text {, enquanto que } 58 \% \\
\text { apresentaram insatisfação com o trabalho. Houve maior } \\
\text { chance de HA entre os docentes mais velhos, sem pós- } \\
\text { graduação, que atuavam também na rede privada, que } \\
\text { apresentavam maior consumo de sal, com } \\
\text { sobrepeso/obesidade, com relação cintura-quadril elevada e } \\
\text { com hipercolesterolemia/hipertrigliceridemia e menor } \\
\text { chance entre os do sexo feminino, que exerciam outra } \\
\text { atividade de trabalho e insatisfeitos com o trabalho. Assim, } \\
\text { o perfil ocupacional docente apresentou influência sobre a } \\
\text { hipertensão. }\end{array}$ & $\begin{array}{l}\text { Este estudo verificou prevalência de } 24,9 \% \text { de HÁ entre } \\
\text { os docentes da educação básica, rede pública de um } \\
\text { município brasileiro. Houve maior chance de HA entre os } \\
\text { mais velhos, sem pós-graduação, atuantes também na rede } \\
\text { privada, com maior consumo de sal, com } \\
\text { sobrepeso/obesidade, com a RCQ elevada e com } \\
\text { hipercolesterolemia/hipertrigliceridemia e menor chance } \\
\text { entre os do sexo feminino, que exerciam outra atividade } \\
\text { de trabalho além da docência e insatisfeitos com o } \\
\text { trabalho. Foi constatado que o perfil ocupacional docente } \\
\text { exerce influência sobre a HA, mesmo após ajuste por } \\
\text { variáveis reconhecidamente associadas à HA. }\end{array}$ \\
\hline $\begin{array}{c}\text { Xavier, P.B., } \\
\text { Garcez, A., Cibeira, } \\
\text { G.H., Germano, A., } \\
\text { \& Olinto, M.T.A. } \\
\text { (2020) }\end{array}$ & Transversal & $\begin{array}{l}\text { A prevalência de hipertensão foi de } 10,3 \% \text {, sendo maior } \\
\text { entre os homens do que entre as mulheres. A hipertensão } \\
\text { mostrou-se associada à elevação da faixa etária, baixa } \\
\text { escolaridade, viver com companheiro, ter sobrepeso ou } \\
\text { obesidade, e ter pelo menos um parente com história de } \\
\text { hipertensão para ambos os sexos. As mulheres com } \\
\text { melhores condições socioeconômicas apresentaram menores } \\
\text { prevalências de hipertensão. }\end{array}$ & $\begin{array}{l}\text { Os principais fatores associados à hipertensão arterial } \\
\text { compreenderam características sociodemográficas, } \\
\text { nutricionais e de história familiar. Ademais, as condições } \\
\text { socioeconômicas demonstraram uma associação com a } \\
\text { ocorrência de hipertensão, principalmente entre as } \\
\text { mulheres. }\end{array}$ \\
\hline Ribeiro, V.E.R., \& & Transversal & A prevalência de HA foi de $24 \%$, sendo $37 \%$ dentre & Verificou-se efeito cumulativo das dimensões da atividade \\
\hline
\end{tabular}




\begin{tabular}{|c|c|c|c|}
\hline $\begin{array}{l}\text { Fernandes, R.C.P. } \\
(2020)\end{array}$ & & $\begin{array}{l}\text { pacientes com idades entre } 35 \text { e } 44 \text { anos, e } 51 \% \text { entre } 45 \text { e } \\
54 \text { anos. O modelo multivariado evidenciou que ativos em } \\
\text { uma dimensão ou em nenhuma tiveram } 62 \% \text { mais HA, e um } \\
\text { valor } 25 \% \text { mais elevado de HA foi observado dentre aqueles } \\
\text { trabalhadores ativos em duas das três dimensões, na } \\
\text { comparação com os ativos nas três / } 2020 \text { dimensões. Sexo } \\
\text { masculino, maior idade (> } 31 \text { anos) e excesso de peso se } \\
\text { associaram à HA, com razões de prevalência de } 1,62,2,10 \text { e } \\
2,26 \text {, respectivamente. }\end{array}$ & $\begin{array}{l}\text { física sobre a ocorrência de HA. Classificar sujeitos ativos } \\
\text { no trabalho ou no ambiente doméstico como inativos ao } \\
\text { se basear apenas na dimensão do lazer pode implicar em } \\
\text { erro metodológico. }\end{array}$ \\
\hline $\begin{array}{c}\text { Santiago, C.D.S., } \\
\text { Travassos, M.C.P., } \\
\text { Sousa, A.M., } \\
\text { Almeida, G.S., \& } \\
\text { Toledo, N.N. (2021) }\end{array}$ & Transversal & $\begin{array}{l}\text { Maioria são mulheres faixa etária entre } 25 \text { e } 44 \text { anos, não } \\
\text { tem cônjuge, ensino superior completo, renda mensal três } \\
\text { salários mínimos. Nos participantes que apresentaram } \\
\text { valores pressóricos elevados durante a medida da PA } \\
\text { observam-se diferenças no grupo que referiu ter: HAS } \\
{[41,7 \%] \text {, diabetes mellitus [25\%], estar obeso ou acima do }} \\
\text { peso [58,3\%]. }\end{array}$ & $\begin{array}{l}\text { Os achados indicam que os servidores públicos com } \\
\text { níveis pressóricos elevados também tinham diagnóstico de } \\
\text { diabetes mellitus, bem como ser obeso ou estar acima do } \\
\text { peso. Os antecedentes familiares de HAS e comorbidades } \\
\text { associadas foram mais expressivos entre os hipertensos. O } \\
\text { sedentarismo foi significativo, tanto entre os hipertensos } \\
\text { como normotensos. Esse estudo reafirma a necessidade da } \\
\text { implementação de estratégias que garantam o manejo de } \\
\text { indivíduos com HAS. }\end{array}$ \\
\hline
\end{tabular}

Fonte: Ramos (2021).

\section{Discussão}

A hipertensão é uma patologia de elevada prevalência na população adulta, isto é, na idade produtiva, o que implica menor produtividade no trabalho (Cesário, 2008; Leão, 2013).

As concessões de benefícios previdenciários por doenças cardiovasculares vêm aumentando. Uma das causas é a alta prevalência da HAS, condição clínica que, embora seja de diagnóstico relativamente fácil e é passível de prevenção e controle, possui expressiva ocorrência de aposentadoria por incapacidade, fato preocupante para o país (Soares et al., 2017).

Apesar dos estudos apontarem o trabalho noturno, insatisfação com o trabalho, baixa remuneração, sobrepeso ou obesidade, sedentarismo e faixa etária como fatores possivelmente associados à hipertensão arterial, são necessários mais estudos sobre o tema — hipertensão e fatores ocupacionais — para ampliar a compreensão dessa problemática.

O controle da hipertensão no ambiente de trabalho pode ser um fator que promova mudanças comportamentais com influência positiva no desenvolvimento das atividades diárias desses trabalhadores. O fator psicológico, mesmo que não apresentado nestes estudos, influencia a maneira de ver, pensar e tratar esta doença. Nesse contexto, a saúde do trabalhador se insere no cenário da saúde pública não apenas ante a significativa morbimortalidade e aos custos para a saúde oriundos de doenças cardiovasculares, mas também diante das possibilidades de ação preventiva e de controle de riscos resultantes da exposição ocupacional a possíveis fatores predisponentes.

Com isso, os achados deste estudo permitem discussões sobre os fatores de risco no ambiente de trabalho e como podem afetar a pressão arterial, como também auxilia os profissionais de saúde, contribuindo assim com a obtenção de maior conhecimento acerca do tema, promovendo prevenção e educação em saúde e melhorando a qualidade da assistência.

Como sugestão para novos estudos sobre o tema consideramos que ter uma pré-avaliação clínica dos trabalhadores, com um intervalo de no mínimo seis meses, entre esta avaliação e o começo do estudo, será um fator relevante para que os dados apurados possam substanciar um trabalho que apresente informações mais claras e conclusivas acerca da correlação ambiente de trabalho x desenvolvimento de hipertensão arterial.

\section{Conclusão}

Apesar da importância do tema hipertensão arterial x correlação com o trabalho/atividades exercidas os artigos analisados não são conclusivos e instigam que seja feita uma análise mais aprofundada sobre o assunto. O fato de identificarem a correlação existente, e que esta traz implicações negativas ao trabalhador e por consequência ao ambiente de trabalho não são argumentos suficientes para que os estudos sejam validados, pois a análise dos indicadores pode estar comprometida pelo fato 
de que a coleta dos dados foi realizada em apenas um momento do estudo. Mais pesquisas sobre a temática são necessárias a fim de obter mais informações sobre o processo saúde/doença dos trabalhadores. Assim, poderão ser implantados programas e ações mais efetivos no ambiente de trabalho, voltados para a qualidade de vida e saúde dos trabalhadores.

Desta forma é correto afirmar que identificar o perfil de hipertensão arterial em trabalhadores, nos mais diversos segmentos, é relevante quando se tem como propósito planejar e programar ações preventivas que possam melhorar a condições de saúde dos trabalhadores.

\section{Referências}

Barreto, M. L., Carmo, E. H., Noronha, C. V., Neves, R. B. B., \& Alves, P. C. (2017). Mudanças dos padrões de morbimortalidade: uma revisão crítica das abordagens epidemiológicas. Physis Rev Saúde Coletiva. 2017,3(1):127-46.

Barroso, W. K. S., Rodrigues, C. S., Bortolotto, L. A., \& Gomes, A. M. (2020). Diretrizes Brasileiras de Hipertensão Arterial - 2020. Arq Bras Cardiol. 2021, 116(3):516-658. https://abccardiol.org/article/diretrizes-brasileiras-de-hipertensao-arterial-2020/

Benjamin, E. J., Virani S. S., Callaway, C. W., Chamberlain, A. M., Chang, A. R., Cheng, S. \& et al. Heart Disease and Stroke Statistics - 2018, Update: A Report From the American Heart Association. Circulation [Internet]. 2018. Volume 137, Issue 12, 20 March 2018, Pages e67-e492 https://doi.org/10.1161/CIR.0000000000000558

Beyea, S. C., Nicoll, L. H. (1998). National Library Of Medicine. National Center for Biotechnology Information. Writing an integrative review. AORN $J$ 1998 Apr,67(4):877-80. 10.1016/s0001-2092(06)62653-7. https://pubmed.ncbi.nlm.nih.gov/9616108/

Broome, M. E. (1993). Integrative literature reviews for the development of concepts. In: Rodgers BL, Knafl KA, editors. Concept development in nursing. Philadelphia: Saunders: 231-50.https://www.researchgate.net/publication/238248432_Integrative_literature_reviews_for_the_development_of_concepts

Buuren, F. van, Horstkotte, D., Knabbe, C., Hinse, D., \& Mellwig, D. H. (2017). Incidence of elevated lipoprotein (a) levels in a large cohort of patients with cardiovascular disease [eletronic version]. Clin Res Cardiol Suppl. 12(Suppl 1):55-59. 10.1007/s11789-017-0087-y.

Carrapato, P., Correia, P., \& Garcia, B. (2017). Health determinants in Brasil: searching for health equity. Articles Saude soc. 26 (3) https://doi.org/10.1590/S0104-12902017170304

Carey, R. M., Muntner, P., Bosworth, H. B., \& Whelton, P. K. (2018). National Library Of Medicine. National Center for Biotechnology Information. Prevention and Control of Hypertension. JACC Health Promotion Series. J Am Coll Cardiol. 72(11):1278-1293. 10.1016/j.jacc.2018.07.008. https://pubmed.ncbi.nlm.nih.gov/30190007/

Carvalho, A. C. S., Maçaneiro, ALS, Santos, E. T. S., Macedo, G. C., Silva, S. B., Flores, G. R., \& Cubo, R. C. P. (2017). Estresse do Enfermeiro no Ambiente de Trabalho e Evolução para Hipertensão Arterial [versão eletrônica]. Anais da $12^{a}$ Semana Acadêmica de Enfermagem das FISA/FUNEC: Boas práticas de Enfermagem e a construção de uma sociedade democrática /v.3, n.3, 2017. ISSN 2525-8141.

Cezário, A. C. (2008). Hipertensão arterial e doenças cardiovasculares como causas de concessão de aposentadoria por invalidez no Brasil. Dissertação de mestrado, Universidade Federal da Bahia, Salvador, BA, Brasil.

Domingues, J. G, Silva, B. B. C., Bierhals, I. O., \& Barros, F. C (2019). Noncommunicable diseases among nursing professionals at a charitable hospital in Southern Brazil. http://scielo.iec.gov.br/scielo.php?script=sci_arttext\&pid=S1679-49742019000200020\&lng=en\&nrm=is\&tlng=en

Forouzanfar, M. H., Liu, P., Roth, G. A., Ng, M., Biryukov, S., Marczak, L., Alexander, L., Estep, K., Abate, K. H., Akinyemiju, T. F., Ali, R., Guzman, N. A., \& et al. National Library Of Medicine. National Center for Biotechnology Information. Global burden of hypertension and syst olic blood pressure of at least 110 to $115 \mathrm{~mm} \mathrm{Hg}, 1990-2015$. JAMA 2017, 317(2):165-82. 10.1001/jama.2016.19043. Erratum in: JAMA. 14,317(6):648.

International Society of Hypertension - ISH (2018). Background information on high blood pressure (hypertension). Recuperado em 18 de outubro, 2021, de https://ish-world.com/background-information/

Leão, A. L. M. (2013). Síndrome Metabólica em funcionários de uma universidade pública: fatores associados e concordância entre critérios de diagnóstico. Dissertação de mestrado, Universidade Federal de Minas Gerais, Belo Horizonte, MG, Brasil.

Lee, L. L., Mulvaney, C. A., Wong, Y. K. Y., Chan, E. S. Y., Watson, M. C., \& Lin, H. H. (2021. Walking for hypertension. Cochrane Database of Systematic

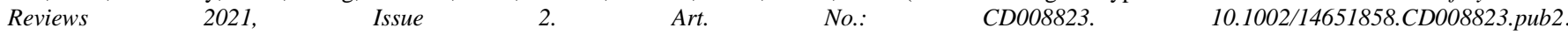
https://www.cochranelibrary.com/cdsr/doi/10.1002/14651858.CD008823.pub2/epdf/full

Malacarne, F. S., Prudente, R. C. S., \& Fonseca, M. R. C. (2017). Shift work: repercussions on health among workers of an Electricity Distribution Company. Perspectivas Médicas, 28(2): 30-36, 10.6006/perspectmed.02042017.5842316589

Ministério da Saúde (2018). Saúde e Vigilância Sanitária. Agência Saúde. 20,7\% da população de Belém diz ter diagnóstico médico de hipertensão. https://antigo.saude.gov.br/noticias/agencia-saude/43125-20-7-da-populacao-de-belem-diz-ter-diagnostico-medico-de-hipertensao

Ministério da Saúde (2019). Secretaria de Vigilância em Saúde. Departamento de Análise em Saúde e Vigilância de Doenças Não Transmissíveis. Vigitel Brasil 2018: vigilância de fatores de risco e proteção para doenças crônicas por inquérito telefônico. Estimativas sobre frequência e distribuição sociodemog'rafica de fatores de risco e proteção para doenças crônicas nas capitais dos 26 estados brasileiros e no Distrito Federal em 2018. Brasília,DF https://portalarquivos2.saude.gov.br/images/pdf/2019/julho/25/vigitel-brasil-2018.pdf 
Research, Society and Development, v. 11, n. 1, e7411124963, 2022

(CC BY 4.0) | ISSN 2525-3409 | DOI: http://dx.doi.org/10.33448/rsd-v11i1.24963

Ministério da Saúde (2020). Saúde e Vigilância Sanitária. Pesquisa Nacional de Saúde 2019. Maioria dos pacientes com hipertensão e diabetes faz acompanhamento de saúde no SUS. https://www.gov.br/pt-br/noticias/saude-e-vigilancia-sanitaria/2020/11/maioria-dos-pacientes-com-hipertensao-e-diabetesfaz-acompanhamento-de-saude-no-sus

Nascimento, J. O. V., Santos, J., Meira, K. C., Pierin, A. M. G., \& Souza-Talarico, J. N. (2019). Shift work of nursing professionals and blood pressure, burnout and common mental disorders. Rev Esc Enferm USP. 53:e03443. http://dx.doi.org/10.1590/S1980-220X2018002103443

Neto, E. M. N., Tania, M. A., \& Sousa, C. C. (2019). Hipertensão Arterial e Diabetes Mellitus entre trabalhadores da saúde: associação com hábitos de vida e estressores ocupacionais [versão eletrônica]. Revista Brasileira de Saúde Ocupacional http://dx.doi.org/10.1590/2317-6369000034218

Pereira, A. L., Bachion, M. M. (2006). Atualidades em revisão sistemática de literatura, critérios de força e grau de recomendação de evidência. Rev Gaúch Enferm. (RS) 2006,27(4):491-8. https://www.researchgate.net/publication/277241223_Atualidades_em_revisao_sistematica_de_literatura_criterios_de_ forca_e_grau_de_recomendacao_de_evidencia

Pimenta, A. M., \& Assunção, A. A. (2016). Estresse no trabalho e hipertensão arterial em profissionais de enfermagem da rede municipal de saúde de Belo Horizonte, MG, Brasil [versão eletrônica]. Revista Brasileira de Saúde Ocupacional Artigo. http://dx.doi.org/10.1590/2317-6369000113515

Polit, D. F., Beck, C. T. (2006). Essentials of Nursing Research: Appraising Evidence for Nursing Practice. 7th Edition, Wolters Kluwer Health/Lippincott Williams \& Wilkins, Philadelphia. https://www.scirp.org/(S(351jmbntvnsjt1aadkposzje))/reference/ReferencesPapers.aspx?ReferenceID=1426133

Ribeiro, V. E. R., \& Fernandes, R. C. P. (2020). Hipertensão Arterial em Trabalhadores: O Efeito Cumulativo das Dimensões da Atividade Física sobre esse Agravo. Arq. Bras. Cardiol. 114 (5) https://doi.org/10.36660/abc.20190065 https://www.scielo.br/j/abc/a/pBQzYM8PRbhjq57FwBRyLpd/?lang=pt

Santiago, C. D. S., Travassos, M. C. P., Sousa, A. M., Almeida, G. S., \& Toledo, N. N. (2021). Pressão Arterial Elevada em Servidores de Universidades Públicas no Norte do Brasil. Revista Cogitare Enfermagem. 26, 986-1005. https://revistas.ufpr.br/cogitare/article/view/74371

Scala, L. C., Magalhães, L. B., \& Machado, A. (2015). Epidemiologia da hipertensão arterial sistêmica. In: Moreira SM, Paola AV, Sociedade Brasileira de Cardiologia. Livro Texto da Sociedade Brasileira de Cardiologia. (2a ed.), Manole, 2015. p. 780-5.

Soares, E. F. G., Pardo, L. S., \& Costa, A. A. S. (2017). Evidence for the work/occupation and systemic hypertension interrelationship: an integrative Review [eletronic version]. Revista Brasileira em Promoção da Saúde, 30(1),102-109. https://www.redalyc.org/articulo.oa?id=40851313014

Souza, M. T., Silva, M. D., \& Carvalho, R. (2010). Integrative review: what is it? How to do it? Original Article • Einstein (São Paulo) 8 (1) https://doi.org/10.1590/S1679-45082010RW1134 https://www.scielo.br/j/eins/a/ZQTBkVJZqcWrTT34cXLjtBx/?lang=en

Vieira, M. R. M., Magalhães, T. A., Silva, R. R. V., Vieira, M. M., Paula, A. M. B., Araújo, V. B., Ferreira, E. F., \& Haikal, D. S. (2020). Hipertensão Arterial e trabalho entre docentes da educação básica da rede pública de ensino. Ciência \& Saúde Coletiva, 25(8):3047-3061, https://doi.org/10.1590/141381232020258.26082018

Whittemore, R, \&Knafl, K, 2005. The integrative review: updated methodology. Methodological issues in nursing research. $J$ Adv Nurs. 2005,52(5):546-53. https://pubmed.ncbi.nlm.nih.gov/16268861/>

Xavier, P. B., Garcez, A., Cibeira, G. H., Germano, A., \& Olinto, M. T. A. (2020). Fatores Associados à Ocorrência de Hipertensão Arterial em Trabalhadores da Indústria do Estado do Rio Grande do Sul, Brasil. Arq. Bras. Cardiol. 117 (3) https://doi.org/10.36660/abc.20190815 